\title{
Hanseníase: conhecimentos e mudanças na vida das pessoas acometidas
}

\author{
H anses's disease: \\ knowledge and changes in the life of wounded people
}

Joyce M azza N unes ${ }^{1}$

Eliany N azaréO Oliveira ${ }^{2}$

N eiva Francenely Cunha Vieira ${ }^{3}$

${ }^{1}$ Centro de Saúde da Família Janival deAlmeida Vieira, Secretaria M unicipal de

SaúdedeFortaleza. Rua

Coelho Garcia 25, Passaré

60861-810 Fortaleza CE.

joycemazza@hotmail.com

${ }^{2}$ Centro deCiências da

Saúde, Universidade

Estadual Vale do Acaraú.

${ }^{3}$ Departamento de

Enfermagem, Faculdade de

Farmácia, Odontologiae

Enfermagem, Universidade

Federal do Ceará.
Abstract Hanses's disease is a millenary illness, characterized as a public health issue. This study aims to verify the knowledge of peoplewith $\mathrm{H}$ ansen's diseaseabout their problem and its treatment and point out possible changes in their lives as a consequence. We conducted interviews with peoplein treatment for multi-bacillary Hansen's diseasein a Basic H ealth U nit of Sobral, Ceará State, in 2005. We realized that the knowledge about the disease is elementary, usually expressed by people's own experience, involving negative feelings. Some bearers showed to have appropriate knowledge according to the literature, acquired through theinternet, books and information from friends and relatives. The main changes in their lives after the diagnosis was related to the use of medicines, as some people related improvement in their symptoms and others worsening, interfering in their daily activities. Key words Hansen's disease, Knowledge, Prejudice
Resumo A hanseníase é uma doença milenar, caracterizada como problema de saúde pública. Este estudo objetiva averiguar o conhecimento de pessoas com hanseníase sobre sua doença e tratamento e investigar possíveis mudanças na vida, como consequência da doença. Realizamos entre vistas com pessoas em tratamento para hanseníase multibacilar em uma U nidade Básica de Saúde do M unicípio de Sobral (CE), em 2005. Percebemos que o conhecimento sobre a doença é rudimentar, geralmente expresso pela própria experiência, envolta de sentimentos negativos. Alguns portadores manifestaram conhecimentos condizentes com a literatura, adquirido através da Internet, livros e informações de profissi onais, amigos e familiares. As principais alterações na vida após o diagnóstico estavam relacionadas ao tratamento medicamentoso, quando alguns relataram melhora dos sintomas e outros, um agravamento, interferindo nas atividades diárias. Palavras-chave Hanseníase, Conhecimento, Preconceito 


\section{Introdução}

A hanseníase é uma doença infecciosa, crônica, causada por uma bactéria denominada micobaterium leprae, que atinge pessoas de todas as idades, principalmente aquelas na faixa etária economicamente ativa, tendo assim grande importância para a saúde pública, devido à sua magnitude e seu alto poder incapacitante ${ }^{1}$.

A transmissão da hanseníase se dá por meio de uma pessoa doente, sem tratamento, que pelas vias áreas superiores (mucosa nasal e orofaringe) elimina o bacilo para o meio exterior, infectando outras pessoas suscetíveis. Estima-se que somente uma parcela da população que entra em contato com a bactéria manifeste a doença, queacomete principalmentea pelee os nervos periféricos, mas também se manifesta de forma sistêmica, comprometendo articulações, olhos, testículos, gânglios e outros órgãos².

0 diagnóstico da hanseníase é realizado essencialmente nos serviços de Atenção Básica de Saúde, por meio do exame dermatoneurológico, com o objetivo de identificar lesões ou áreas de pele com alteração de sensibilidade e/ou comprometimento denervos periféricos. No momento do diagnóstico, é feita a classificação operacional do caso de hanseníase, com base no número de lesões cutâneas, de acordo com os seguintes critérios: paucibacilar (PB), pessoas com até cinco lesões de pele, emultibacilar (MB), pessoas com mais de cinco lesões de pele². Essa classificação operacional visa ao tratamento ambulatorial com o esquema PQT/OM S (poliquimioterapia), que deve ser supervisionado pelo profissional de saúde. Para os casos paucibacilares, o tratamento dura seis meses e, para os multibacilares, dura doze meses.

A hanseníaseéuma doença muito antiga, que tem uma terrível imagem na história ena memória da humanidade, pois desde tempos remotos tem sido considerada uma doença contagiosa, mutilante eincurável, ocasionando rejeição, discriminação e exclusão do doente na sociedade. As pessoas acometidas pela hanseníase foram confinadas e tratadas em leprosários durante muitos anos, o que deu origem ao estigma da doença e ao preconceito contra o doente ${ }^{1}$.

0 estigma ligado à hanseníase ocorre desde tempos imemoráveis, sendo por isso uma condição complexa, que exige um processo de mudança gradativo através dos anos. Nesse sentido, muitas ações foram e ainda estão sendo desenvolvidas com o objetivo de amenizar esse estigma. Uma dessas ações foi a mudança do nome lepra para hanseníase, mas nem todos os países adotaram esta nomenclatura. 0 Brasil teve a iniciativa pioneira de substituir oficialmente o termo lepra por hanseníase, contribuindo para a redução do preconceito e o estigma que envolve a doença3.

Grande esforço global tem sido empregado, sob orientação da Organização M undial de Saúde(OM S), com o objetivo de eliminar a hanseníase como problema de saúde pública. O riginalmente, esse objetivo deveria ter sido atingido em 2000. No entanto, o Brasil vem mantendo uma média de 47 mil novos casos de hanseníase anualmente no último quinquênio, com um parâmetro alto de endemicidade, especialmente nas regiões N orte, N ordeste e Centro-Oeste 4 .

No estado do Ceará, a hanseníase é de alta endemicidade e está em expansão. N osso estado ocupa o décimo lugar do país em número de casos e terceiro do Nordeste. Dos 184 municípios do estado, doze $(6,5 \%)$ foram selecionados pelo M inistério da Saúde no ano de 2007 como prioritários para intervenção 5 . Um desses municípios prioritários é Sobral, localizado na zona do sertão centro-norte do estado do Ceará, onde muitas ações têm sido desenvolvidas no sentido de eliminar a doença, como o incentivo ao diagnóstico precoce, a capacitação das equipes de saúde da família e a descentralização da assistência aos doentes.

A experiência de uma das autoras como enfermeira da Estratégia Saúde da Família (ESF) do Município de Sobral (CE), prestando assistência de enfermagem a pessoas acometidas pela hanseníase, permitiu observar que, mesmo na atualidade, apesar dos significativos avanços, a hanseníase continua sen do uma doença estigmatizante, que acarreta transtornos físicos e psicológicos às pessoas em tratamento. Assim, nos motivamos a estudar a hanseníase, nos aproximando das pessoas acometidas por ela com os objetivos de averiguar o conhecimento de pessoas em tratamento para hanseníase sobre sua doença e tratamento e investigar possíveis mudanças na vida dessas pessoas, após o diagnóstico da doença.

A profundar estudos sobreesta temática éinstigante e, ao mesmo tempo, desafiador, uma vez queinquieta, nos dias dehoje, a maioria dos profissionais de saúde que se depara com ela.

Acreditamos que os achados do estudo poderão contribuir para a melhoria da assistência prestada as pessoas com hanseníase e suas famílias, especialmente na Estratégia Saúde da Família, pois a equipe de saúde da família está mais 
próxima ao contexto em que estão inseridas essas pessoas, podendo atuar no sentido de ampliar esse conhecimento e auxiliar no enfrentamento da doença.

\section{Métodos}

N este estudo, utilizamos uma abordagem qualitativa. Os sujeitos foram pessoas em tratamento para hanseníase multibacilar em uma Unidade Básica de Saúde (UBS) localizada na sede do município de Sobral (CE). A escolha por pessoas em tratamento para hanseníase multibacilar se deu pelo fato de que, nessa classificação operacional, estão as formas mais graves da doença, cujo tratamento é mais prolongado, podendo causar maior prejuízo na qualidade de vida das pessoas ${ }^{6}$.

Para a coleta de informações, realizamos a técnica degrupo focal, queéum tipo de entrevista em grupo que val oriza a interação e a comunicação entre os participantes da pesquisa, a fim de gerar informações. Isso significa que, em vez do pesquisador pedir a cada pessoa para responder a uma pergunta por vez, as pessoas são estimuladas a falar umas com as outras, a perguntar, a trocar histórias e experiências e comentar sobre os pontos de vista umas das outras?

O convite para participar do grupo focal foi entregue pelos agentes comunitários de saúde (ACS) a todas as dezesseis pessoas que realizavam tratamento para hanseníase multibacilar nesta UBS no mês de agosto de 2005. Entretanto, somente oito pessoas, seis homens e duas mulheres, compareceram ao local e dia agendado para o grupo focal e aceitaram participar do estudo.

O grupo se reuniu na sala de Educação em Saúde desta UBS, local tranquilo, privativo elivre de ruídos externos, já que o ambiente onde ocorre o grupo focal é um aspecto importante e deveser cuidadosamente planejado ${ }^{7}$. Esteencontro durou duas horas e discussão foi norteada por dois pontos de investigação: (1) a hanseníase e o seu tratamento e (2) a vida depois do diagnóstico de hanseníase. Buscando-se estabelecer um diálogo aberto com os participantes, utilizamos uma linguagem adequada a estes sujeitos. A fala de cada participante foi gravada com autorização dos mesmos.

Para obtermos dados pessoais, clínicos e socioeconômicos dos participantes, utilizamos um formulário contendo as seguintes questões: idade, sexo, situação conjugal, escolaridade, profissão/ocupação, renda familiar, condições de moradia e tempo de tratamento, com a intenção de situar as pessoas portadoras de hanseníase no contexto social em que estão inseridas.

Os princípios éticos e legais do estudo foram respeitados. 0 projeto de pesquisa foi aprovado pelo ComitêdeÉtica e Pesquisa do Centro deCiências da Saúde da Universidade Estadual Vale do Acaraú (UVA), sendo norteado pela Resolução n ${ }^{\circ}$ 196/96 do Conselho Nacional de Saúde, sobre pesquisa envolvendo seres humanos ${ }^{8}$. Os participantes do estudo foram esclarecidos sobre os objetivos da pesquisa, sendo garantido o sigilo de suas identidades e tendo o direito de desistir a qualquer momento de participar do estudo, em qualquer um de seus estágios. Os pesquisadores apresentaram a cada participante um termo de consentimento livre e esclarecido, que foi assinado por todos ou pelos seus responsáveis.

Para identificar os sujeitos do estudo, preservando seu anonimato eilustrando os depoimentos, atribuímos a cada um deles o nome de uma pedra preciosa, pois assim como as pedras preciosas, cada pessoa reflete um brilho eluz própria.

Para análise dos resultados, transcrevemos na íntegra as fitas gravadas com as fal as de cada participante durante o grupo focal e realizamos os seguintes passos: (a) leituras exaustivas do relatório obtido, com o intuito de compreender e apreender o sentido das informações; (b) condensação das informações a partir de cada pergunta; (c) identificação em cada item dos pontos comuns e agrupamento das similaridades, permitindo a construção de categorias deanálise; (d) discussão das categorias, com vistas aos objetivos propostos e (e) análise reflexiva das respostas significativas para o estudo, com o intuito deaprofundamento do objeto. Estes passos foram propostos para a organização de informações de teor qualitativo ${ }^{9}$, que utilizamos em nosso estudo por considerarmos a melhor opção para sintetizar e discutir os resultados encontrados.

\section{Análiseediscussão dos resultados}

\section{Conhecendo os sujeitos do estudo}

Os sujeitos do estudo foram pessoas em tratamento de hanseníase multibacilar, sendo seis homens e duas mulheres, que estavam numa faixa etária compreendida entre quinze e oitenta anos; dois eram solteiros, um separado e cinco eram casados. Em relação à escolaridade, um era analfabeto, seis tinham o ensino fundamental e um concluiu o ensino superior. A renda familiar era inferior a um salário mínimo para dois sujei- 
tos, de dois salários mínimos para três sujeitos, de três salários para dois sujeitos e de até quatro salários mínimos para um deles. As ocupações/ profissões dos participantes do estudo eram estudante, desempregado, aposentado, catador de lixo, zelador, professor, vendedor ambulante e serral heiro. Estas pessoas realizavam tratamento para hanseníase multibacilar há pelo menos quatro meses.

\section{Cenas do grupo focal}

No dia da realização do grupo focal na UBS, as pessoas em tratamento para hanseníase convidadas foram chegando e ficaram esperando o início da reunião no pátio da UBS, aguardando o momento de iniciar o encontro, que estava marcado para as oito horas da manhã. Quando tudo ficou preparado (som, gravador, microfone, cadeiras), os convidados entraram na ampla Sala de Educação em Saúde e o encontro pôde ser iniciado. Dispomo-nos numa "roda" com os oito sujeitos que compareceram, seis homens e duas mulheres.

Satisfação expressa nos rostos, postura de cidadãos. A sensação era decumprimento de um compromisso de interesse pessoal. Demonstravam familiarização com o serviço. Todos vestiam roupas apropriadas e em harmonia com sua situação financeira, cabelos penteados e olhares de expectativa em relação à reunião. A percepção que tínhamos era de que todos eram de casa.

0 encontro iniciou com a apresentação individual dos presentes. Os participantes não ficaram tímidos, falaram abertamente, sem a necessidade de interferirmos para estimular a conversa. As falas foram se estendendo com a participação crescente dos envolvidos e refletiam o impacto da doença na vida de cada um deles. Surgiram várias narrativas interessantes que foram gravadas com a permissão dos mesmos.

Percebemos que aquele momento parecia fazer parte de seu tratamento, quando se sentiram à vontade para sugerirem que houvesse outros momentos assim, em que pudessem se reunir e conversar sobre hanseníase, entre si e com os profissionais de saúde. Assim, compreendemos que as pessoas acometidas pela hanseníase têm necessidade de expor o que sentem, de falar sobre a doença e trocar experiências com outros portadores e com os profissionais de saúde, 0 que muitas vezes não é possível acontecer nas consultas individuais mensais para a administração da dose supervisionada.
Conhecimento sobre a doença e o tratamento

A partir desta temática, em que o questionamento objetivavaidentificar o conhecimento dos portadores de hanseníase sobre a doença e o tratamento, observamos que em geral este conhecimento sobre a doença pouco está relacionado com as informações fornecidas pelos profissionais de saúde. Os sujeitos do estudo manifestaram como conhecimento sobre hanseníase sua própria experiência vivenciada. Em geral, esse conhecimento é expresso por várias experiências e sentimentos negativos. Os portadores falam com propriedade da doença sentida, da doença experienciada, da doença como algo próximo e que se manifesta em seus corpos. 0 experienciar da doença os habilita a descreverem sinais, sintomas, dores, medos e ansiedades.

Um participante do estudo relatou não compreender as informações fornecidas pelos profissionais de saúde. A linguagem técnica que em muitos casos se faz presente na relação entre os profissionais e portadores da hanseníase vai de encontro às possibilidades de apreensão dessas informações pelos portadores. Esta fragilidade no processo de cuidado impulsiona os portadores para o aprofundamento de um conhecimento/reconhecimento da doença, tendo-se em vista singularidades vivencias por cada um. N estecontexto, a definição da doença se iguala às sensações e manifestações vividas:

A hanseníase é uma malfeitoria na pessoa. Eu me sinto bem fazendo o tratamento, tem que tomar aqueles comprimidos fortes, mas tem que aguentar, eu fico tonto, parece que bebi cachaça. M efalaram não sei o quê, não sei o quê, queeu não entendo, só os médicos e enfermeiras entendem, maseu, pelo menos não entendo. Eu sinto dor numa perna e apresentei calombos pelo corpo, mas hoje estou livre, graças a Deus. (Q uartzo)

0 que eu sei équeessa doença émuito nojenta, muito pesada, é muito maltratadeira, maltrata a gente demais, acaba com a gente, me dá quentura no corpo todo, nos braços, nas pernas, sinto azia, me sinto mal. Sinto muita coisa dessa doença. 0 tratamento vai bem, a gente toma aqueles comprimidos depois do almoço, quando vem receber no posto, toma os seis comprimidos grandes de uma vez. (Citrino)

Eu sei que é uma doença muito pesada, judeia muito com a gente, a gente toma o medicamento e tem uma reação mais pesada, eu tenho sofrido muito esses dias, é muita quentura no corpo, dores nas pernas, dormência na boca, eu tenho sofrido, 
mas estou tentando até o fim! 0 tratamento é assim: eu estou com nove meses de tratamento, que é de um ano, eu tomo dois comprimidos todos os dias, aí tô vivendo, pelejando. (Topázio)

É importante relatar que, em relação ao tratamento medicamentoso, nenhum portador de hanseníase que participou deste estudo soube informar os nomes dos medicamentos utilizados. Entretanto, sabiam o tempo de duração do tratamento, a quantidade administrada na dose mensal supervisionada e na dose diária e alguns efeitosindesejados.

Em estudo desenvolvido com adolescentes portadores de hanseníase em Sobral, foi possível observar que os mesmos, ao expressar seu conhecimento sobre a doença, deram ênfase às manifestações clínicas, modo de transmissão e complicações, detectando-se em algumas falas déficit deconhecimento dealguns adolescentes ${ }^{10}$.

Reforçando nossas análises, outro estudo sobre a hanseníase observou que o conhecimento da doença pelos pacientes é rudimentar, a comunicação entre o pessoal do centro de saúde estudado e os pacientes é precária, pois parcela significativa da população pesquisada de doentes não entendem as informações prestadas pelo centro de saúde ou as consideram incompletas ${ }^{11}$.

Reconhecemos que alguns portadores de hanseníase que participaram do nosso estudo, além de manifestarem conhecimentos embasados nas próprias experiências vivenciadas, relataram conhecimentos condizentes com a literatura, tendo obtido essas informações em fontes como livros, I nternet ou amigos que já tiveram a doença, pois sentiam necessidade de conhecer melhor a doença da qual eram portadores, reivindicando inclusive uma melhor comunicação com a equipe de saúde. As falas ilustram essa assertiva:

Eu sei que a hanseníase é uma bactéria que ataca os nervos ou em forma de manchas. Se for paucibacilar, o tratamento é de seis meses e, se for multibacilar, o tratamento é de um ano. A multibacilar, a gente toma aqueles comprimidos horrorosos. 0 tratamento é com os comprimidos poliquimioterápicos, que juntos tentam combater a bactéria, mas não dão conta. Eu fui atrás de descobrir o que era hanseníase pela Internet e pelos livros, mas os profissionais de saúde deveriam dar essas informações pra gente. (Esmeralda)

A minha hanseníase se manifestou através de manchas, eu senti como uma agulha furando meu pé. Eu conversei com um amigo que teve a doença e ele me disse que dava muita coisa, dor nos joe Ihos, nervos e ao pisar, comigo aconteceu isso, te- nho realizado o tratamento direito, como manda a medicina e graças a Deus estou bem. A hanseníase é uma doença que pega pelo bafo. (Rubi)

0 fato de essas pessoas terem procurado obter mai ores informações através de outras fontes de informações não se constitui um fato negativo. Pelo contrário, na era da globalização, da informatização, énatural queaquelas pessoas que possuem maiores condições socioeconômicas e/ ou culturais procurem obter maiores informações nos meios de comunicação acessíveis, como Internet e livros. Entretanto, a grande maioria das pessoas portadoras de hanseníase não se enquadra nesse perfil, conforme observamos neste estudo.

Através das entrevistas, reconhecemos que estes sujeitos possuem algum conhecimento sobre a doença, mas ainda existe a necessidade de ampliar essas informações. N esse sentido, as ações de educação em saúde se tornam imprescindíveis, o diálogo entre os envolvidos deve ser utilizado como a principal ferramenta. As pessoas devem ser reconhecidas como sujeito portador de um saber que deve ser valorizado, embora possa ser diverso do saber técnico-científico ${ }^{12}$.

Mudanças na vida

após o diagnóstico de hanseníase

Esta temática foi estruturada a partir do se gundo ponto de investigação: a vida depois do diagnóstico de hanseníase. As respostas foram relacionadas principalmenteaos efeitos do tratamento da hanseníase. Alguns sujeitos do estudo relataram melhora na sua vida após a descoberta da hanseníase, pois final mente obtiveram uma conclusão diagnóstica e, com o início do tratamento, houve melhora considerável dos sintomas da doença, amenizando dores e dormências no corpo, trazendo-Ihes sensação de bem-estar:

Antes de descobrir que estava com hanseníase, eu sentia muitas dores e era muito ruim, mas fui tomando o medicamento e fui melhorando, as dores melhoraram e eu me sinto melhor agora, mas eu quero terminar logo o tratamento, porque eu não quero ficar com essa doença dentro de mim. (Diamante)

É, minha vida mudou, está muito mudada. No começo, me deu muita preocupação, assim que eu soube quetava com essa doença, mas depois queeu estou me tratando, tomando os remédios direito, me sinto bem melhor. (Rubi)

Pra mim o que mudou foi que antes eu vivia preocupado em saber o que eu tinha e sentia muitas coisas, me sentia ruim, mas com os medica- 
mentos eu melhorei me sinto bem melhor, estou bem. (Ametista)

Através das falas, percebemos que um sujeito do estudo manifestou preocupação ao ser diagnosticado com hanseníase; outro sujeito preocupou-se em concluir logo o tratamento, pois não queria permanecer portador da doença. Estes achados nos remetem à conotação negativa da hanseníase.

Alguns participantes do estudo relataram que, após o diagnóstico de hanseníase, sua vida mudou drasticamente. Essas mudanças são associadas diretamente aos efeitos indesejáveis do tratamento medicamentoso, como por exemplo, o escurecimento da pele. Por ser uma doença incapacitante, alguns portadores de hanseníase manifestaram que a doença lhes causa moderadas dores por todo o corpo, pondo em risco a harmonia de sua vida, impedindo-os de trabalhar e realizar tarefas diárias, impondo limitações às suas atividades, acarretando verdadei ras mudanças em suas vidas, como também a diminuição das atividades de lazer e sociais:

A conteceu que eu sinto muitas dores nos ossos, perdi até o jeito de viver, porque não pude mais trabalhar como eu vivia trabalhando, satisfeito, e não pude mais, a bicha maltrata muito a gente, judeia comigo, sinto quentura no corpo, me dá dores no corpo todo e eu não posso viver direito. (Quartzo)

A lepra mudou muito a minha vida. Eu não gosto detá mais no meio demuita gente, evito ficar no meio das pessoas, de pegar em crianças, eu não gosto não. Sinto muitas dores no corpo, faço muita coisa, mas não me sinto bem, faço minhas atividades durante o dia equando chega a noite meu corpo lateja de dor mesmo. (Safira)

Pra mim, mudou muito a minha vida, porque eu fiquei doente depoisque comecei esse tratamento, porque eu não tinha o que tenho agora, eu não posso andar muito, não posso levantar peso, não posso escrever muito, não posso ficar muito tempo sentada, nem em pé, mudou drasticamente a minha vida. Hoje não consigo dormir direito, adquiri muitas incapacidades que antes eu não tinha e as dificuldades para trabal har e andar. M eus amigos me perguntam por que engordei, mudei de cor, mas às vezes eu não digo não. (Esmeralda)

Estudos já apontaram a hanseníase como um problema que gera sofrimento social e impacto no cotidiano das pessoas acometidas ${ }^{10,13}$. Em adolescentes portadores de hanseníase, a sintomatologia da doença, os efeitos colaterais da medicação e as reações interferem significantemente no seu cotidiano, visto que o lazer e os afazeres domésticos são afetados ${ }^{10}$.
Estudo que aval iou a qualidade de vida em paciente com hanseníase concluiu que esta doença causa grande prejuízo para a vida diária das pessoas e as relações interpessoais, provocando sofrimento que ultrapassa a dor e o mal-estar vinculados ao prejuízo físico, o que causa grande impacto social e psicológico. Esse prejuízo na qualidade de vida está associado demaneira mais importanteàs formas mais graves da doença, como a multibacilar, reação hansênica e incapacidades físicas ${ }^{6}$.

É importante ressaltar que um sujeito do estudo utilizou a nomenclatura lepra para relatar que a hanseníase mudou a sua vida, enquanto esta nomenclatura já está em desuso no Brasil. Este mesmo sujeito manifestou diminuição das atividades sociais, não apenas em decorrência das dores físicas, mas também por opção própria, o que pode levar a um isolamento social. Outro achado que merece destaque foi o relato de ocultação da doença por um sujeito do estudo, mesmo ao ser indagado sobre mudanças na sua aparência física, em consequência do tratamento medicamentoso. Estes achados podem ser atribuídosao estigma epreconceito em relação à hanseníase, que existem desdetempos remotos, quando a doença ainda era denominada de lepra.

Profissionais de saúde que prestam assistência a pessoas acometidas pela hanseníase acreditam que o estigma é o principal responsável pelo preconceito e isolamento social dos doentes de hanseníase ${ }^{14}$. N este sentido, estudo abordando a consulta de enfermagem a pessoas em tratamento para hanseníase na Estratégia Saúde da Família em Sobral (CE) percebeu que os enfermeiros valorizam principalmente dois fatores durante as consultas: as orientações sobre prevenção de incapacidadesea atenção para tentativa de minimizar o estigma social que envolve a hanseníase ${ }^{15}$.

\section{Considerações finais}

N este estudo, buscamos desvelar os contextos que envolvem as pessoas em tratamento de hanseníase, identificamos seus conhecimentos e as repercussões da doen ça em suas vidas; fez-senecessário acolhermos todas as informações obje tivas e subjetivas e idéias do grupo, valorizando os sentimentos, crenças e percepções manifestas.

Constatamos que o conhecimento sobre a doença é rudimentar e em geral expresso pela própria experiência vivenciada, envolta de sentimentos negativos. Entretanto, alguns portadores manifestaram conhecimentos condizentes com a literatura, adqui ridos através de uma bus- 
ca solitária por informações na I nternet, livros e informações de amigos e familiares. Destacamos a necessidade da utilização pelos profissionais de saúde de uma linguagem clara e adequada ao perfil da clientela, para que as informações em saúde sejam efetivamente apreendidas. Ações de educação em saúde são imprescindíveis na assistência a essa clientela, reconhecendo os saberes existentes e transformando-os, incentivando a troca de experiência entre os envolvidos ea construção compartilhada de conhecimentos.

Como principais alterações na vida após o diagnóstico de hanseníase, manifestaram a meIhora dos sintomas com o início do tratamento; alguns, ao contrário, relataram o aparecimento ou agravamento sinais e sintomas físicos desagradáveis, influenciando sua qualidade de vida e causando sentimentos de impotência.

A utilização da nomenclatura lepra, a ocultação da doença e o isolamento social por parte dos portadores de hanseníase também foram achados deste estudo.

Percebemos que a hanseníase não passa despercebida na vida das pessoas, ela deixa sua marca (física ou emocional), seja por tempo limitado durante o tratamento ou por um período mais longo, como no caso das sequelas físicas. Nesse sentido, imprescindível é o apoio dos profissionais de saúde a essas pessoas, prestando uma atenção integral.

Necessário também é criar espaços de escuta das pessoas com hanseníase, onde possam manifestar suas crenças, dúvidas e conversar sobre a doença e sobre sua vida. I dentificamos no estudo uma ferramenta importante no objetivo de atingir esse ideal, a realização de "rodas de conversa" com pessoas que realizam tratamento para hanseníase e os profissionais de saúde, possibilitando a troca de saberes e experiências entre os envolvidos, disseminando também as informações acerca da doença e seu tratamento, esclarecendo dúvidas frequentes eauxiliando-as no enfrentamento da hanseníase.

Esperamos que o estudo possa contribuir no aprimoramento da assistência prestada pelos profissionais de saúde a essa clientela, especialmente na Estratégia Saúde da Família, por estar mais próxima a essas pessoas e suas famílias, podendo desenvolver suas ações voltadas para o contexto que as envolve.

\section{Colaboradores}

JM N unes eEN Oliveira trabalharam na concepção teórica, coleta de informações e análise dos resultados; NFC Vieira trabalhou na concepção teórica e redação final do texto. 


\section{Referências}

1. Brasil. M inistério da Saúde. Secretaria de Políticas de Saúde. Departamento de Atenção Básica. H anseníase: Atividades de controle e manual de procedimentos/ área técnica de dermatologia. Brasília (DF): M inistério da Saúde; 2001.

2. Brasil. M inistério da Saúde. Secretaria de Atenção à Saúde. Departamento de Atenção Básica. Caderno de Atenção Básica no 21. Vigilância em Saúde: Dengue, Esquistossomose, Hanseníase, M alária, Tracoma e Tuberculose. $2^{\underline{a}}$ ed. rev. Brasília (DF): Ministério da Saúde; 2008.

3. Queiroz MS, Puntel MAA. Endemia hansênica: uma perspectiva multidisciplinar. Rio de Janeiro: Fiocruz; 1997.

4. Brasil. M inistério da Saúde. Secretaria de Atenção à Saúde. Departamento de Atenção básica. Informe da Atenção Básica № 042 . A responsabilidade da Atenção Básica no diagnóstico precoce da hanseníase. Braślia (DF): M inistério da Saúde; 2007.

5. Governo do Estado do Ceará. Hanseníase: doença em expansão no Ceará. Informe Epidemiológico. Hanseníase. Fortaleza: Governo do Estado do Ceará; 2008.

6. Martins BDL, Torres F, Oliveira M LW. Impacto na qualidade de vida em pessoas com hanseníase: correlação do Dermatology life quality index com diversas variáveis da doença. An. bras. dermatol. 2008; 83(1):39-43.

7. Kitzinger J. Grupos focais com usuários e profissionais da atenção à saúde. In: Pope $\mathrm{C}, \mathrm{M}$ ays $\mathrm{N}$, organizadores. Pesquisa qualitativa na atenção à saúde. $2^{\mathrm{a}}$ ed. Porto Alegre: Artmed; 2005.

8. Guathier JH M, Cabral IE, Santos I, Tavares CM M Pesquisa em Enfermagem: novas metodologias aplicadas. Rio de Janeiro: Guanabara Koogan; 1998.

9. M inayo CS. Os muitos Brasis: saúde e população na década de 80. 2ae ed. São Paulo: Hucitec; 1999.
10. Ponte KMA, Ximenes Neto FRG. Hanseníase: a realidade do ser adolescente. Rev. Bras. Enferm. 2005; 58(3):296-301.

11. Queiroz MS, Carrasco MAP. 0 doente de hanseníase em Campinas: uma perspectiva antropológica. Cad Saude Publica 1995; 11(3):479-490.

12. Alves VS. U m modelo de Educação em Saúde para - Programa Saúde da Família: pela integralidade da atenção e reorientação do modelo assistencial. Interface (Botucatu) 2005; 9(16):39-52.

13. Lira GV. Avaliação da Ação Educativa em Saúde na perspectiva compreensiva: 0 caso da hanseníase [dissertação]. Fortaleza (CE): U niversidade de Fortaleza; 2003.

14. Pereira AJ, Helene LM, Pedrazzani ES, M artins CL, Vieira CSC A. Atenção Básica de Saúde e a assistência em $\mathrm{H}$ anseníase em serviços de saúde de um Município do Estado de São Paulo. Rev. Bras. Enferm. 2008; 61(Esp.):716-726.

15. Freitas CASL, Silva Neto AV, Ximenes Neto FRG, Albuquerque IMN, Cunha ICKO. Consulta de Enfermagem ao portador de Hanseníase no Território da Estratégia Saúde da Família: percepções de enfermeiro e pacientes. Rev. Bras. Enferm. 2008; 61(Esp.):757-763.

Artigo apresentado em 14/11/2008

Aprovado em 16/02/2009

Versão final apresentada em 02/03/2009 